\title{
Fate of algebraic many-body localization under driving
}

\author{
Heiko Burau $\odot,{ }^{1}$ Markus Heyl, ${ }^{1}$ and Giuseppe De Tomasi ${ }^{2,3}$ \\ ${ }^{1}$ Max Planck Institute for the Physics of Complex Systems, 01187 Dresden, Germany \\ ${ }^{2}$ T.C.M. Group, Cavendish Laboratory, JJ Thomson Avenue, Cambridge CB3 OHE, United Kingdom \\ ${ }^{3}$ Department of Physics, University of Illinois at Urbana-Champaign, Urbana, Illinois 61801-3080, USA
}

(Received 18 August 2021; accepted 16 November 2021; published 3 December 2021)

\begin{abstract}
In this work we investigate the stability of an algebraically localized phase subject to periodic driving. First, we focus on a noninteracting model exhibiting algebraically localized single-particle modes. For this model we find numerically that the algebraically localized phase is stable under driving, meaning that the system remains localized at arbitrary frequencies. We support this result with analytical considerations using simple renormalization group arguments. Second, we inspect the case in which short-range interactions are added. By studying both the eigenstate properties of the Floquet Hamiltonian and the out-of-equilibrium dynamics in the interacting model, we provide evidence that ergodicity is restored at any driving frequencies. In particular, we observe that for the accessible system sizes localization sets in at driving frequency that is comparable with the many-body bandwidth and thus it might be only transient, suggesting that the system might thermalize in the thermodynamic limit.
\end{abstract}

DOI: 10.1103/PhysRevB.104.224201

\section{INTRODUCTION}

Understanding the breakdown of ergodicity in the quantum realm is an active and fast growing front of research, motivated by recent developments of controllable quantum simulations which allows one to access out-of-equilibrium dynamics [1,2]. In particular, many-body localization (MBL) describes the paradigm of ergodicity breaking in quantum phases of matter, generalizing the concept of Anderson localization to the interacting case [3-6].

An MBL phase is described by an emergent form of integrability, i.e., the existence of a complete set of quasilocal integrals of motion (LIOMs) [7-10]. These LIOMs are adiabatically connected to the integrals of motion of the noninteracting model and thus are typically exponentially localized. As a consequence, transport is absent, some memory of the local structure of its initial state is retained during the quantum evolution, and the interactions between the LIOMs allow a slow information propagation through the system [11-13]. The MBL phase should be distinguished from an ergodic or thermal one, in which eigenstates are chaotic and are expected to obey the eigenstate thermalization hypothesis (ETH) [14-17].

Several works have studied the stability of the MBL phase in the case of interacting one-dimensional short-range systems subject to strong disorder [12,18-22]. Recently, the possible existence of MBL has been shown in systems in which its single particles are power-law localized, dubbed algebraic

Published by the American Physical Society under the terms of the Creative Commons Attribution 4.0 International license. Further distribution of this work must maintain attribution to the author(s) and the published article's title, journal citation, and DOI. Open access publication funded by the Max Planck Society.
MBL [23,24]. Algebraic MBL generalizes the paradigm of power-law localization to the interacting case and therefore its LIOMs develop algebraically decaying tails. An immediate consequence of having power-law localized LIOMs is that the bipartite entanglement entropy after a quantum quench has an unbounded algebraic growth in time [23,25,26], unlike the case with exponentially localized single particles for which the entanglement propagates logarithmically slowly $[11,13]$. Furthermore, an increasing amount of efforts have been devoted to study the fate of MBL under periodic driving [27-35]. In Refs. $[27,28]$ it was shown that at high frequency an MBL phase evades the fate of thermalization remaining localized, while at sufficiently low frequency the system is delocalized. As a consequence of the robustness of an MBL phase under driving, it has been possible to explore novel nonequilibrium quantum phases of matter, i.e., discrete time crystals which are characterized by a spontaneous breaking of discrete time translation symmetry [29-33].

The aim of this work is to investigate the stability of an algebraic MBL under driving. In particular, we periodically drive (Floquet) a system of spinless fermions with long-range hopping known to have algebraically localized single-particle wave functions $\left[|\psi(x)| \sim 1 /|x|^{\alpha}\right][36-41]$.

First, we study the stability of a noninteracting algebraically localized phase by numerically inspecting several probes to distinguish a localized from an extended phase. We conclude that for the noninteracting case the localized phase does not break down under periodically driving. We support this claim by analytical considerations based on strongdisorder renormalization group techniques. Next, we focus on the interacting case. By studying both the eigenstate properties of the Floquet Hamiltonian and the out-of-equilibrium dynamics, we provide numerical evidence that the algebraic MBL phase breaks down under driving. We surrogate this conjecture with simple analytical considerations. Thus, ergodicity is restored at any finite drive's frequency in the 

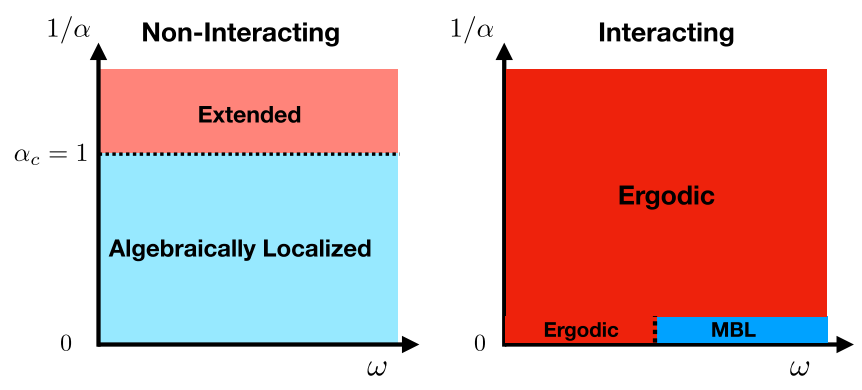

FIG. 1. Schematic phase diagram of an algebraically localized phase under driving. $\alpha$ is the rate of decay of the hopping amplitudes and $\omega$ is the frequency of the driving. The noninteracting localized phase is robust under driving (left). Instead, ergodicity is restored at any driving frequency for the interacting case (right). In the limiting case $\alpha \rightarrow \infty$ (short range), the system undergoes an MBL transition at a finite driving frequency.

thermodynamic limit. This result might imply the nonexistence of time crystals with algebraically localized LIOMs $[31,32,42-44]$. Figure 1 summarizes schematically the phase diagram of an (non)interacting algebraically localized phase under driving.

The rest of the work is organized as follows. In Sec. II we introduce both the noninteracting and the interacting models and we discuss the different probes that have been used to detect localization and delocalization. Section III A is devoted to probe the stability of a noninteracting case. We support these findings using a renormalization group approach, which is explained in Sec. III B. Finally, in Sec. IIIC we study numerically the stability of an algebraic MBL phase subject to driving.

\section{MODEL AND METHODS}

We consider a periodically driven system of spinless fermions on a one-dimensional lattice with long-range hopping

$$
\begin{aligned}
H(t)= & \sum_{i \neq j}\left(J_{i j} c_{i}^{\dagger} c_{j}+\text { H.c. }\right)+[1+\delta(t)] \sum_{i} h_{i} n_{i} \\
& +V \sum_{i} n_{i} n_{i+1},
\end{aligned}
$$

where $J_{i j}=J_{j i}=\mu_{i j} / \sqrt{1+|i-j|^{2 \alpha}}$ describes a disordered long-range hopping involving all pairs of sites, with an algebraically decaying amplitude with respect to their distance according to an exponent $\alpha>0[36-41,45,46]$. Quenched hopping disorder is given by $\left\{\mu_{i j}\right\}$, which is uniformly distributed between $[-1,1]$, and onsite disorder is added by $\left\{h_{i}\right\}$ which are random fields uniformly distributed between $[-W, W] . V$ is the interaction strength and $L$ is the number of sites and we indicate with $N=L / 2$ (half-filling) the number of particles.

We choose a binary driving protocol of period $T$, i.e., $\delta(t)=-\delta$ for $0<t<T / 2$ and $\delta(t)=+\delta$ for $T / 2<t<T$ and $\delta(t+n T)=\delta(t), n \in \mathbb{Z}$. Our particular choice of driving the external potential is motivated by the experimental ease of implementation. We find that, qualitatively, our results for the noninteracting case do not depend on the choice of the driven term. As an energy reference we define the hopping unit $J=\sqrt{\frac{1}{2 L} \sum_{i \neq j}\left\langle J_{i j}^{2}\right\rangle}$, where $\langle\cdot\rangle$ indicates the average over disorder and by $\omega_{D}=2 \pi / T$ we denote the driving frequency in the following.

We now briefly summarize the dynamical phase diagram of the nondriven Hamiltonian $H(\delta=0)$ in Eq. (1). The noninteracting case without driving $(V, \delta=0)$, referred as power-law banded random matrix (PLBRM), is known to have a metalinsulator transition at $\alpha=1$ from an extended $(\alpha<1)$ to an algebraically localized phase $(\alpha>1)$ [41,47,48]. Thus, for $\alpha>1$ the single-particle orbitals are algebraically localized, meaning that they possess power-law decaying tails around their centers of localization $i_{c}, \psi(i) \sim\left|i-i_{c}\right|^{-\alpha}$. In this work, we are interested in the robustness of this localized phase under driving, therefore, we mostly consider $\alpha>1$.

As interactions are turned on $(V>0)$, the nondriven system goes through an MBL transition as a function of the disorder strength $W[23,48]$. For $V=1$ and $\alpha=3$, the critical point has been estimated to be $W_{c} \approx 3$, ergodic for $W<W_{c}$ and localized for $W>W_{c}$. The MBL phase of $H$ in Eq. (1) is described by a complete set of LIOMs $\left\{\tau_{i}\right\}_{i}^{L},{ }^{1}$ which have algebraically decaying tails, i.e, $\operatorname{Tr}\left[\tau_{i} A_{j}\right] \sim 1 /|i-j|^{\alpha_{1}}$, where $A_{j}$ is a generic local observable with support at site $j$ [23].

It is important to point out that the question whether MBL is stable in higher dimensions or for long-range Hamiltonians is still under debate [49-66]. Recent experiments in fairly large system sizes and for relevant timescales have shown evidence of an MBL phase for both two-dimensional systems and long-range Hamiltonians [67-70]. However, in quantumavalanche theory the proposed mechanism for delocalization invokes the existence of rare entropic thermal bubbles, which will have effect at very long timescales and large system sizes [49-51,71,72].

We will numerically show that the algebraic MBL phase is vastly unstable against external driving. This fragility appears to be independent of the driving frequency, although partially accompanied with finite-size effects depending on the domain of frequency. As a result, we observe a qualitative difference with respect to the behavior from short-ranged, or a "conventional" MBL phase under driving, in which the LIOMs are exponentially localized. For the latter, it is believed that a system-size independent critical driving frequency $\omega_{c}$ exists, above which the system remains localized [27,28,34].

Throughout this paper, numerical results were obtained using free-fermion techniques for the noninteracting case and exact diagonalization for the interacting one. In order to establish the existence of a localized phase, we use a variety of probes, each giving insights to the dynamical phase structure from different facets. In particular, for each quantity we average over at least $10^{2}$ random configurations and over energy spectrum.

In the remainder of this section we describe all utilized probes in detail.

$$
{ }^{1}\left[H, \tau_{i}\right]=0 \forall i .
$$




\section{A. Level-spacing statistics}

The distribution of level spacing has been found to be a useful probe to distinguish an ergodic phase from an MBL one $[17,73,74]$. In an ergodic phase at infinite temperature, the level spacing is expected to be distributed as the one of a random matrix belonging to the same universality class of the Hamiltonian, namely, the Gaussian orthogonal ensemble (GOE). However, in an MBL phase, due to the existence of LIOMs, energy levels are weakly correlated and the level spacings are Poisson distributed.

Specifically, the degree of proximity to a random matrix ensemble can be extracted from the distribution of level spacing through the so-called $r$ value, or gap-ratio parameter, which is defined as

$$
r=\left\langle\min \left(\delta_{n} / \delta_{n+1}, \delta_{n+1} / \delta_{n}\right)\right\rangle,
$$

where $\delta_{n}$ are level spacing of two consecutive level energies and $\langle\cdot\rangle$ refers to the disorder and spectrum average [73,74]. The $r$ value in the ergodic phase is $r_{\mathrm{GOE}} \approx 0.531$, while it is $r_{\text {Poisson }}=2 \log 2-1$ in a localized one.

For the periodically driven system in Eq. (1), we consider the quasienergies of the Floquet Hamiltonian $H_{\mathrm{F}}$ defined by

$$
e^{-i H_{\mathrm{F}} T}=U(T)=\mathcal{T} e^{-i \int_{0}^{T} d t^{\prime} H\left(t^{\prime}\right)},
$$

which serves as generator for the discrete translation in time of period $T .^{2}$

\section{B. Energy propagation}

In driven systems the energy is not conserved during time evolution and at any discrete time step an extensive amount of energy is pumped up in the system. Thus, in an ergodic phase the long-time asymptotic steady state is expected to be a structureless infinite-temperature state. However, in an MBL phase the expected heating is effectively prevented at high driving frequencies. This can be understood from the emergence of an extensive number of locally conserved quantities, which effectively hinder the system to exchange energy beyond a characteristic localization length [27,28,34].

In order to study the energy absorbed through the quantum time evolution, we define the dimensionless parameter

$$
\epsilon(\tau)=\frac{\langle\psi(\tau)|H(\tau=0)| \psi(\tau)\rangle-E_{\min }}{E_{\max }-E_{\min }},
$$

where $\tau=t / T$ and $|\psi(\tau)\rangle$ is the time-evolved state, starting from the ground state of $H(\tau=0)$. Here, $E_{\min }$ and $E_{\max }$ refer to the lowest and highest eigenvalues of $H(\tau=0)$, respectively. In an ergodic phase in the thermodynamic limit the asymptotic long-time value of $\epsilon(\tau \rightarrow \infty)=\frac{1}{2}$ (middle of the spectrum), while in an MBL phase $\epsilon(\tau \rightarrow \infty)<\frac{1}{2}$.

\section{Entanglement entropy}

The MBL transition can be understood in terms of a rearrangement of the local structure of entanglement in the system $[18,19,21]$. In particular, the half-chain bipartite entanglement

\footnotetext{
${ }^{2} \mathcal{T}$ denotes the time-ordered product.
}

entropy (EE) measures the amount of entanglement through the cut and can be used both to detect the MBL transition and to characterize the two phases. The $\mathrm{EE}(S)$ is defined through the half-partition reduced density matrix $\rho_{L / 2}$, where the sites belonging to one-half of the system have been traced out:

$$
S=-\operatorname{Tr}\left(\rho_{L / 2} \ln \rho_{L / 2}\right) .
$$

In an ergodic phase at infinite temperature, the eigenstates behave like random vectors and, therefore, the EE reaches the so-called Page value $S_{\text {Page }}=L / 2 \log 2-\frac{1}{2}[75] .^{3}$

For the out-of-equilibrium dynamics, we compute the EE following a quantum quench starting from the Néel state $|\psi(0)\rangle=\prod_{i}^{N} c_{2 i}^{\dagger}|0\rangle$. In an ergodic system driven in time, $S(\tau)$ increases up to the value of an infinite-temperature thermal state $S_{\text {Page }}=L / 2 \log 2-\frac{1}{2}$. In an MBL phase, on the other hand, $S(\tau)$ is known to saturate to an extensive value which is not thermal $[11,13]$, thus we expect $\lim _{t \rightarrow \infty} S(\tau) / S_{\text {Page }}<1$.

Furthermore, we compute the EE for the eigenstates of the effective Hamiltonian $H_{\mathrm{F}}$, which will have a volume law $S \sim O(L)$ in the ergodic phase and a subvolume scaling in the localized one $\lim _{L \rightarrow \infty} S / L \rightarrow 0$ [76].

\section{Inverse partition ratio}

Finally, for the noninteracting case $(V=0)$, we compute the inverse partition ratio (IPR) [77] for the single-particle eigenfunctions of the Floquet Hamiltonian $H_{\mathrm{F}}$, which is defined as

$$
\operatorname{IPR}\left(\epsilon_{v}\right)=\sum_{i}\left|\phi_{v}(i)\right|^{4},
$$

where $i$ indicates the lattice site and $\phi_{v}(i)$ refers to the singleparticle wave function with single-particle energy $\epsilon_{v}$. The IPR separates a delocalized phase from a localized one from its scaling with $L$, IPR $\sim O\left(L^{-1}\right)$ in a delocalized phase and IPR $\sim O\left(L^{0}\right)$ in a localized one.

We emphasize that in driven systems the density of states (DOS) is constant, up to finite-size fluctuations, due to the lack of energy ordering, therefore, we can safely average $\operatorname{IPR}\left(\epsilon_{\nu}\right)$ over all energies.

\section{RESULTS}

\section{A. Noninteracting case}

In this section, we inspect the eigenstate properties of the Floquet Hamiltonian $H_{\mathrm{F}}$ for the noninteracting case. In this case, $H_{\mathrm{F}}$ is still a quadratic Hamiltonian $H_{\mathrm{F}}=\sum_{i, j} h_{i j}^{\mathrm{F}} c_{i}^{\dagger} c_{j}$. Thus, we can simply focus on the single-particle energies $\left\{\epsilon_{\nu}\right\}$ and eigenstates $\left\{\phi_{\nu}\right\}$ of $H_{\mathrm{F}}$.

We start our analysis by inspecting the level statistics of the single-particle Floquet Hamiltonian $h^{\mathrm{F}}$. Figure 2 shows the level-spacing parameter $r$ computed with $\left\{\epsilon_{\nu}\right\}$ as a function of the driving frequency $\omega_{D}=2 \pi / T$. As expected, at low frequencies $\left(\omega_{D} \ll J\right)$ the system is more delocalized than higher ones $\left(\omega_{D} \gg J\right)$. Indeed, at low frequencies and relatively small system sizes $L$, the $r$ value is close to the one

\footnotetext{
${ }^{3}$ It is important to point out that, however, also fully ergodic states can reach the Page value [88].
} 

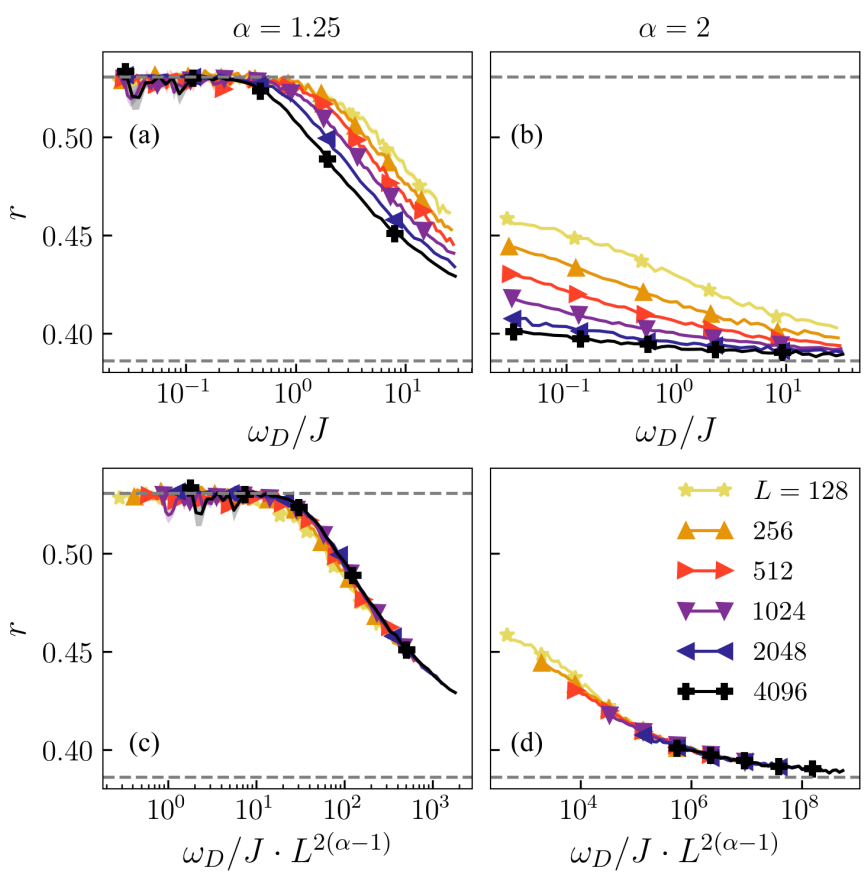

FIG. 2. The $r$-statistics parameter for the quasieigenenergies of the noninteracting model with for $\alpha=1.25$ (a), (c) and $\alpha=2$ (b), (d) for several system sizes $L$. (c), (d) Show the collapse of $r$ with $L, r=r\left(\omega / J L^{2(\alpha-1)}, \delta, W\right)$. In all panels $W=6$ and $\delta=0.5$. The horizontal dashed lines indicate the GOE value $\approx 0.531$ and the Poissonian one $\approx 0.386$.

of a random matrix $\left(r_{\mathrm{GOE}} \approx 0.52\right)$ [see Fig. 2(a)]. However, an overall scaling towards localization (Poissonian statistics) with increasing $L$ is visible in Figs. 2(a) and 2(b). As shown in Figs. 2(c) and 2(d), we found the scaling law which describes the crossover from delocalization to localization with increasing $L$ :

$$
r=r\left(\omega / J L^{2(\alpha-1)}, \delta, W\right)
$$

We note that we checked the validity of this scaling law also for other values of $\alpha>1$. Thus, only in the $L$-dependent frequency regime $\omega_{D} \ll J L^{-2(\alpha-1)}$ the single-particle wave function is delocalized. Importantly, the above scaling law breaks down at the metal-insulator transition $(\alpha=1)$ of the PLBRM. Indeed, for $\alpha<1$ we expect level energies to show repulsion, as visible in Fig. 3(a), where the $r$ value is shown as a function of $\alpha$ at fixed frequency for several system sizes $L$. Finite-size scaling analysis shows that the phase diagram of the PLBRM, delocalized for $\alpha<1$ and localized for $\alpha>1$, remains unchanged under driving [see inset in Fig. 3(a)].

Thus, we conclude that the localized phase is robust under driving, meaning that for $\alpha>1$ and at any finite frequency $\omega_{D}$ the spectrum of the single-particle quasienergy remains Poissonian in the thermodynamic limit $(L \rightarrow \infty)$. For the sake of completeness, we further confirm this result by inspecting the IPR in Eq. (6) for the single-particle wave functions $\left\{\phi_{\nu}\right\}$ of the Floquet Hamiltonian, which quantifies their degree of localization in real space. In Fig. 4 we present the averaged IPR for two values of $\alpha$ and fixed $\omega_{D} / J=0.1$. In agreement with the analysis of the $r$-level statistics, the IPR converges to
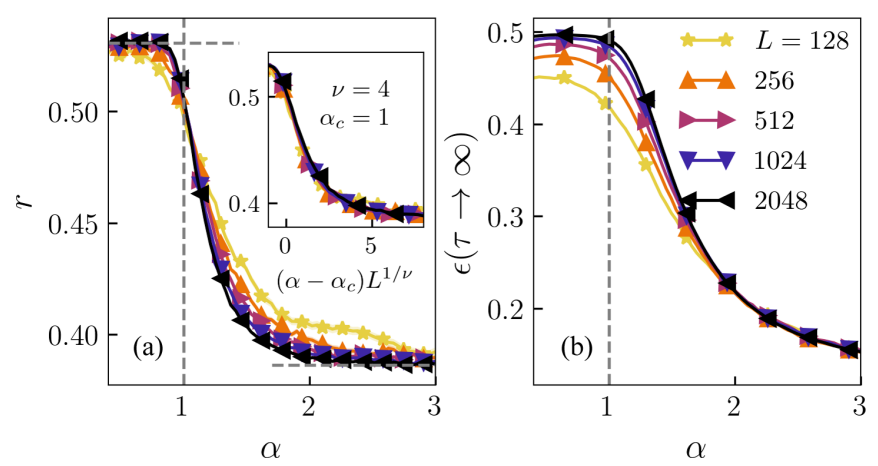

FIG. 3. The $r$ statistics of quasieigenenergies (a) and long-time saturation value of the absorbed energy density $\epsilon(\tau)$ (b) as function of $\alpha$ for the noninteracting case. The system is driven with a frequency $\omega_{D}=J(\alpha)$ in (a) and $\omega_{D}=J(\alpha) / 10$ in (b), both with $W=6$ and $\delta=0.5$. The inset in (a) shows the finite-size scaling proving evidence of the transition at $\alpha=1$. In (a) the horizontal dashed lines indicate the GOE value $\approx 0.531$ and the Poissonian one $\approx 0.386$. In both panels, the vertical dashed line at $\alpha=1$ markers the delocalization-localization transition for the PLBRM.

a finite value with $L\left[\lim _{L \rightarrow \infty} \operatorname{IPR}(L)>0\right]$ and therefore the system is localized [see inset in Fig. 4(a) for $\alpha=2.5$ ].

We complete our analysis for the noninteracting case by investigating the behavior of two dynamical indicators, the energy transport and the information propagation quantified by $\epsilon(\tau)$ in Eq. (4) and $S(\tau)$ in Eq. (5), respectively. As explained in Sec. II, the energy density $\epsilon(\tau)$ is computed starting from the ground state of $H(\tau=0)$ and for the EE from the Néel state $\prod_{i}^{N} c_{2 i}^{\dagger}|0\rangle$.

As expected, in the localized phase the system does not heat up to infinite temperature, meaning that $\epsilon(\tau)<\frac{1}{2}$ and the saturation value of EE is subvolume $\left[\lim _{L \rightarrow \infty} S(\infty) / S_{\text {Page }} \rightarrow\right.$ 0] [76], as one can see in Fig. 5. In particular, we study the long-time saturation value of $\epsilon(\tau)$ as function of the parameter $\alpha$. As one can see in Fig. 3(b), $\epsilon(\infty) \approx \frac{1}{2}$ for $\alpha \leqslant 1$, meaning that in the delocalized phase energy diffuses to its maximum value.

To summarize, by inspecting several indicators, i.e., level spacing, IPR, entanglement, and transport properties, we have
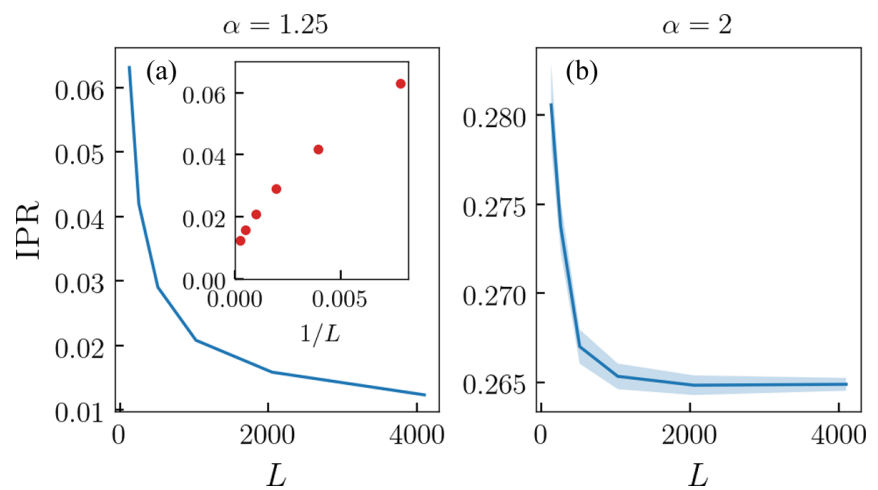

FIG. 4. The inverse partition ratio (IPR) as function of $L$ for the single-particle wave functions for $\alpha=1.25, \alpha=2$ in (a) and (b), respectively. The inset in (a) shows a finite-size scaling of IPR with $L$, indicating that $\lim _{L \rightarrow \infty} \operatorname{IPR}(L) \sim O\left(L^{0}\right)$. 

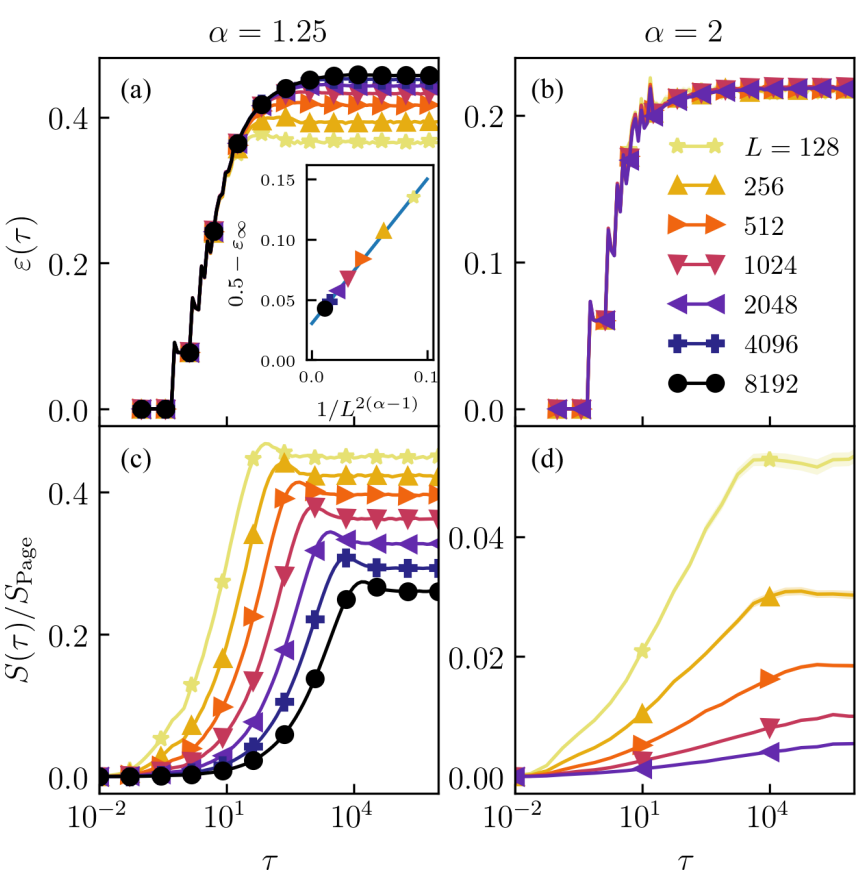

FIG. 5. Absorbed energy density $\epsilon(\tau)$ in Eq. (4) in (a) and (b) and the half-chain bipartite entanglement entropy in (c) and (d) as function of the period driving period $\tau$. The inset in (a) shows the finite-size scaling of the saturation value at asymptotic large times of $\epsilon(\tau) . S(\tau)$ is normalized with the value of the entanglement entropy of a random state $S_{\text {Page }}=L / 2 \log 2-1 / 2$.

provided numerical evidence that the noninteracting localized phase remains localized once the system is driven in time.

\section{B. Driven flow equations}

In the previous section, we have shown numerically that the noninteracting localized phase is robust under driving. We now present an analytical argument supporting our results.

For this purpose, we closely follow a recent renormalization group (RG) approach [47] which uses flow equations [78] to diagonalize a static Hamiltonian. After briefly reviewing this RG approach, we extend it to the periodic driving case $\delta(\tau) \neq 0$, and show that the driving becomes irrelevant in the RG sense. Thus, we will conclude that external driving does not destroy the phase structure of the noninteracting case, in agreement with our numerical results.

In Ref. [47] Quito et al. have shown that the diagonalization of Eq. (1), with $V, \delta(\tau)=0$, can essentially be decomposed into a sequence of two-body problems, i.e., a successive decimation of bonds, whereby the level-spacing statistics of the entire Hamiltonian is maintained. This strictly holds as long as $\alpha>\frac{1}{2}$ and becomes asymptotically exact in the limit $\alpha \rightarrow \infty$ [47]. Let us consider a single RG step or, in other words, the decimation of a bond between two lattice sites $i, j$. Here it is convenient to define new variables $\mathcal{J}=$ $J_{i j}$ and $x=h_{i}-h_{j}$. Now, the Wegner-Wilson flow [79-82] defines a continuous scale $\Gamma$ together with unitary transformations $U(\Gamma)=\mathcal{T}_{\Gamma} \exp \left[\int^{\Gamma} d \Gamma^{\prime} S\left(\Gamma^{\prime}\right)\right]$ which diagonalizes the problem in the limit $\Gamma \rightarrow \infty . S(\Gamma)$ is the instantaneous generator $S(\Gamma)$ and satisfies the condition $\left[H_{0}(\Gamma), S(\Gamma)\right]=$
$H_{1}(\Gamma)$, where $H_{0}=\frac{x}{2}\left(n_{i}-n_{j}\right)$ refers to the diagonal and $H_{1}=\mathcal{J} c_{i}^{\dagger} c_{j}+$ H.c. to the off-diagonal part of the two-body problem (for details see Ref. [47]). Here, the explicit $\Gamma$ dependency is dropped for simplicity. Applying $U(\Gamma)$ to the Hamiltonian or, equivalently, solving the Heisenberg equation $\partial_{\Gamma} H(\Gamma)=[S(\Gamma), H(\Gamma)]$, with $H(\Gamma)=H_{0}(\Gamma)+H_{1}(\Gamma)$, leads to a continuous decay of the off-diagonal matrix elements $H_{1}(\Gamma)$ such that $H(\Gamma)$ becomes diagonal for $\Gamma \rightarrow \infty$. With the explicit form of $S=\mathcal{J} x c_{i}^{\dagger} c_{j}-$ H.c. the above Heisenberg equation can be straightforwardly expressed by the following coupled differential (flow) equations:

$$
\begin{aligned}
\frac{\partial}{\partial \Gamma} \mathcal{J} & =-x^{2} \mathcal{J}, \\
\frac{\partial}{\partial \Gamma} x & =4 x \mathcal{J}^{2},
\end{aligned}
$$

where $\mathcal{J} \rightarrow 0$ for $\Gamma \rightarrow \infty$ with arbitrary initial conditions. A characteristic RG timescale on which $\mathcal{J}$ decays is given by $\tau_{\Gamma}=1 / r^{2}$ with $r^{2}=4 \mathcal{J}^{2}+x^{2}$ being an integral of motion with respect to $\Gamma$ in Eq. (8). The timescale $\tau_{\Gamma}$ sets an order of the RG steps beginning with the largest $r_{\max }^{2}$. The fact that $r^{2}$ is conserved gives rise to an intuitive picture of the renormalization process: each RG step moves one point (bond) within the $x$-J plane towards the $x$ axis on a circle of radius $r$. Thereby, $x_{\Gamma \approx \tau_{\Gamma}}^{2} \rightarrow x_{\Gamma=0}^{2}+4 \mathcal{J}_{\Gamma=0}^{2}$ gets renormalized. As a side note, it should be mentioned that a renormalization of adjacent bonds affected by the flow can be neglected here as the distribution $P(\mathcal{J})$ behaves invariant under the full RG flow [47]. The above picture of renormalization therefore connects the initial distribution $P_{\Gamma=0}(\mathcal{J}, x)$ to the final one $\left[P_{\Gamma \rightarrow \infty}(x)\right]$. The latter is equivalent to the level-spacing distribution $P(s)$ in the limit $x \rightarrow 0$.

With this picture in mind, of the RG accumulating decimations on a circular shell, two phases can be immediately identified: (i) For an initially uniform distribution $P_{\Gamma=0}(\mathcal{J}, x) \sim$ const, one obtains an extended phase, exhibiting level repulsion, i.e., $P_{\Gamma \rightarrow \infty}(x) \sim x$. This is because the number of accumulated decimations $N_{\text {dec }}$ within a shell $[r-$ $d r, r]$ is $N_{\text {dec }} \propto 2 \pi r d r$ in this case [47]. (ii) For any kind of short-ranged distribution $P_{\Gamma=0}(\mathcal{J}, x) \sim e^{-\mathcal{J} / \xi}$, where $\xi \ll$ $r_{\max }$ is a characteristic length scale, the RG leads to a localized phase since $P_{\Gamma \rightarrow \infty}(x) \sim$ const (no level repulsion).

The initial marginal distribution $P(\mathcal{J})$ in our system consists of two regimes separated by a critical $\mathcal{J}_{c} \sim \frac{1}{L^{\alpha}}$ [47], set by the typical hopping amplitude at maximum distance $L$ :

$$
P_{\Gamma=0}(\mathcal{J}) \sim \begin{cases}1 /\left(J^{1+1 / \alpha}\right), & \mathcal{J}>\mathcal{J}_{c} \\ L^{\alpha}, & \mathcal{J}<\mathcal{J}_{c} .\end{cases}
$$

As described above by means of the RG, these two regimes map to two different phases: For $\mathcal{J}<\mathcal{J}_{c}$, the distribution in Eq. (9) is constant, leading to level repulsion, while for $\mathcal{J}>\mathcal{J}_{c}$ it is concentrated near $\mathcal{J}=0$ and thus avoids level repulsion. At the crucial scale of the mean level spacing $\bar{\delta} \sim \frac{1}{L}$ (bandwidth can roughly be considered size independent), the normalized critical $j_{c}$ defined by $j_{c}=\mathcal{J}_{c} / \bar{\delta} \sim \frac{1}{L^{\alpha-1}}$ then gives a critical point at $\alpha=1$. 


\section{Dynamical extension}

In this section we derive a dynamical extension of Eqs. (8) that accounts for a periodic driving term $\delta(t) \neq 0$ in the Hamiltonian. As a simplification we start with a harmonic driving ansatz,

$$
H_{0}(t)=\frac{x}{2}\left(1+\delta e^{i \Omega t}+\text { H.c. }\right)\left(n_{i}-n_{j}\right)
$$

where $\Omega$ denotes a driving frequency and $\delta$ a dimensionless driving strength. A time-dependent change of basis is generally given by $H^{\prime}(t)=e^{-S(\tau)} H(t) e^{S(\tau)}-i \partial_{t} S(\tau)$, with the Schrieffer-Wolff (SW) generator $S(\tau)=-S^{\dagger}(t)$. In order to continuously dampen the off-diagonal hopping terms of $H_{1}$, we use the following generator:

$S(\tau)=\mathcal{J} x\left[c_{i}^{\dagger} c_{j}+\left(\frac{\delta}{1-\omega} c_{j}^{\dagger} c_{i}-\frac{\delta}{1+\omega} c_{i}^{\dagger} c_{j}\right) e^{i \Omega t}\right]-$ H.c.

where $\omega=\Omega / x$ represents a normalized driving frequency. For simplicity, this generator is chosen such that it does not create a time dependence for the off-diagonal hopping terms during the flow. It can be seen that the continuous change of basis with $S(\tau)$ can be expressed in closed form by the following, "driven" flow equations, where we omit the explicit $\Gamma$ dependencies:

$$
\begin{aligned}
\frac{\partial}{\partial \Gamma} \mathcal{J} & =-x^{2} \mathcal{J}\left(1+2 \delta^{2} \frac{\omega}{1-\omega^{2}}\right), \\
\frac{\partial}{\partial \Gamma} x & =4 x \mathcal{J}^{2} \\
\frac{\partial}{\partial \Gamma}(x \delta) & =-4 h \delta \mathcal{J}^{2} \frac{1}{1-\omega^{2}} .
\end{aligned}
$$

A few considerations are in order. (i) We neglected higherharmonic terms $\propto \delta^{4}$, which naturally arise from a time dependent $S(\tau)$. (ii) The driving strength $\delta$ enters quadratically and therefore explains the need for a relatively large value of $\delta=\frac{1}{2}$ to see significant effects from driving in our numerics. (iii) In the infinite frequency limit $\omega \rightarrow \infty$ the flow equations just reduce to the static ones, consistent with the universal scaling law of Eq. (7).

For extraction of the phase structure we are only interested in the behavior at large RG times $\tau_{\Gamma}$ since they correspond to small values of $x$ of the level spacing. This implies that large RG times also correspond to high values of $\omega=\Omega / x$. The above set of equations thus can be further simplified by considering only terms up to leading order in $1 / \omega$ :

$$
\begin{aligned}
\frac{\partial}{\partial \Gamma} \mathcal{J} & =-x^{2} \mathcal{J}\left(1-\frac{2 \delta^{2}}{\omega}\right), \\
\frac{\partial}{\partial \Gamma} x & =4 x \mathcal{J}^{2}, \\
\frac{\partial}{\partial \Gamma}(x \delta) & =0 .
\end{aligned}
$$

Within this approximation we find a first-order correction of the integral of motion $r^{2}$ to $\tilde{r}^{2}=4 J^{2}\left(1+\frac{2 \delta^{2}}{\omega}\right)+x^{2}$. Hence, a typical level spacing from the static case gets scaled by $\frac{2 \delta^{2}}{\omega}$. By identifying $\tau_{\Gamma} \approx 1 / x^{2}$, the driving correction becomes manifestly irrelevant in the course of the RG, $\frac{2 \delta^{2}}{\omega} \approx \frac{1}{\sqrt{\tau_{\Gamma}}} \frac{2 \delta^{2}}{\Omega}$.
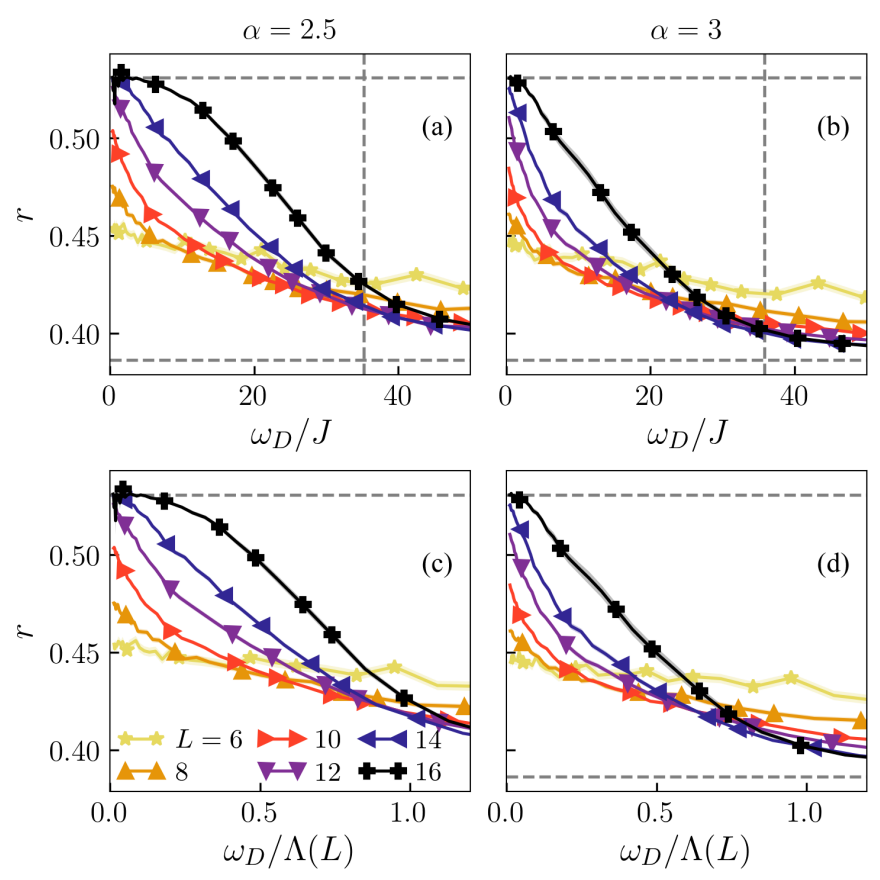

FIG. 6. $r$ statistics for the interacting case $(V=1)$ as a function of the driving frequency $\omega_{D}$, for two values of the hopping decaying rate $\alpha=2.5,3$ in (a) and (b), respectively. The dashed horizontal lines indicate the GOE $r$ value $\approx 0.531$ and the Poissonian one $\approx$ 0.386 , whereas the vertical lines indicate the spectral width $\Lambda(L=$ 16). In (c) and (d), we show the $r$ value as function of $\omega_{D} / \Lambda(L)$. In all panels $W=6$ and $\delta=0.5$.

As a result, the noninteracting algebraically localized phase is robust against driving.

\section{Algebraic MBL}

Having established that the noninteracting algebraic phase is stable under periodic driving, we now turn to the interacting case by setting $V=1$ in Eq. (1). With the aim to understand the robustness of an algebraic MBL phase, we fix the parameters $\alpha$ and $W$ of $H$ in Eq. (1) such that the nondriven system is localized $[48,76,83]$. The numerical results have been computed using exact diagonalization techniques, thus, we will be limited by relatively small systems $L \in[6,16]$. However, by studying several probes to distinguish an ergodic phase from an MBL one, even if affected by finite-size effects, we provide numerical evidence that at any finite frequency, ergodicity is expected to be restored in the thermodynamic limit.

As well as for the noninteracting case, we start our analysis by inspecting the spectrum of the effective Hamiltonian $H_{\mathrm{F}}$ in Eq. (3). Figure 6 shows the $r$ value as function of the driving frequency $\omega_{D}$, for several $L$. A clear trend towards delocalization $(r \approx 0.531)$ with increasing $L$ is visible in Figs. 6(a) and 6(b). The $r$ values change their behavior at high frequency $\omega_{D} \gg J$, where a frequency-independent plateau is formed close to the Poissonian value $r \approx 0.386$. This plateau belongs to the finite-size perturbative regime, in which the system is close t]o the nondriven case $U(T) \approx$ $\mathbb{I}-\frac{i T}{2}[H(T / 2)+H(T)$, which disappears in the thermodynamic limit. Generically, a driving localized phase remains 

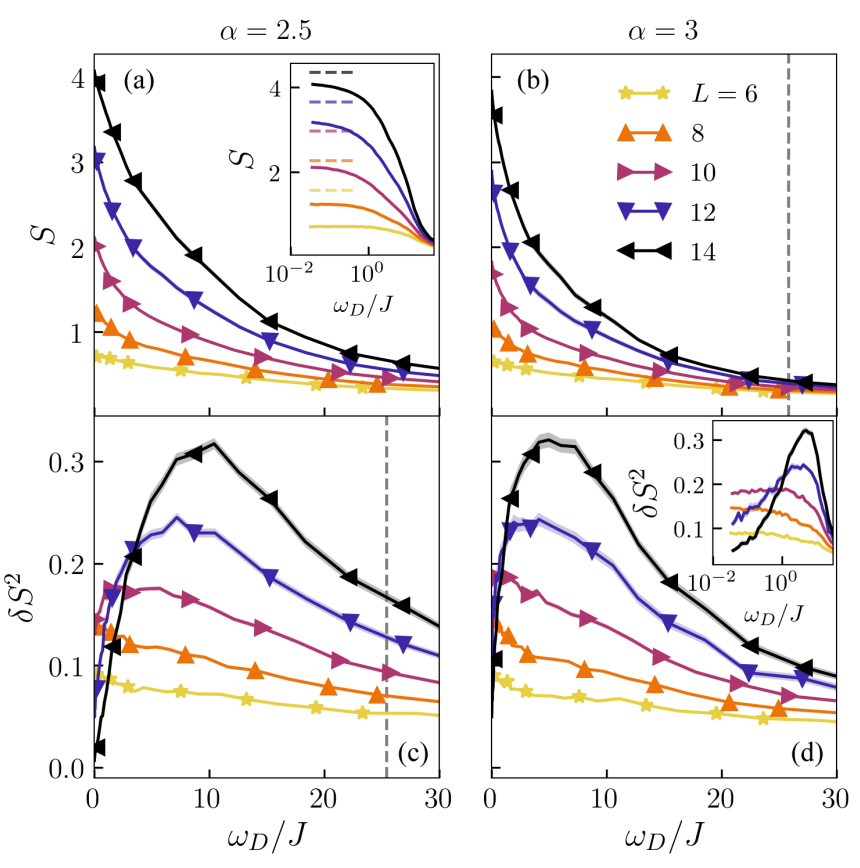

FIG. 7. The averaged entanglement entropy $S$ of the Floquet Hamiltonian for $V=1$ and the variance of the entanglement entropy $\delta S^{2}$ in (a) and (b) and (c) and (d), respectively. The inset in (a) shows $S$ at smaller frequencies for several $L$ with respective Page values. $\delta S^{2}$ at smaller frequencies for $\alpha=3$ is shown in the inset of (d). The dashed vertical lines indicate the bandwidth $\Lambda(L)$.

localized at enough high frequencies. In particular, this will happen if the driving frequency $\omega_{D}$ exceeds the many-body bandwidth $\Lambda(L)$ of the energy spectrum of the nondriven Hamiltonian [27]. We compute the averaged density of states of the nondriven Hamiltonian and using a Gaussian fit we extrapolate its typical bandwidth $\Lambda(L)$ (vertical dashed line in Fig. 6 for the largest $L$ ).

Thus, for $\omega_{D} \geqslant \Lambda$ our numerical result cannot be used to understand the dynamical phase diagram of $H(t)$. By safely ignoring the high-frequency regime $\left(\omega_{D} \geqslant \Lambda\right)$, the $r$ value of $H_{\mathrm{F}}$ shows a clear tendency towards delocalization (rigid spectrum), allowing us to claim that ergodicity might be restored in the thermodynamic limit. This is visible in Figs. 6(c) and $6(\mathrm{~d})$, where the $r$ value is showed as function of $\omega_{D} / \Lambda(L)$, providing evidence that the critical frequency $\omega_{c}$ for which localization sets on, scales as $\omega_{c} \sim \Lambda(L) \sim \sqrt{L}$.

With the aim to further support the claim of delocalization of an algebraic MBL phase under driving, we study the entanglement properties of the eigenstates of the Floquet Hamiltonian $\left(H_{\mathrm{F}}\right)$. Figure 7 shows the bipartite EE averaged over disorder and eigenstates and its fluctuations $\delta S^{2}$ as function of the driving frequency $[19,21]$. The EE has a similar behavior as the $r$-level statistics parameter, meaning that low-frequency EE reaches the ergodic values [see inset in Fig. 7(a)] and then the curves indicate a shift to delocalization (volume-law scaling) before approaching the natural cutoff $\Lambda$. Furthermore, we study fluctuations around the mean value $S$, which are quantified by the variance $\left(\delta S^{2}\right)$ over random samples and eigenstates $[19,21]$. At low frequencies $\delta S^{2}$ decays with $L$ in agreement with the ETH [see inset in Fig. 7(d)].
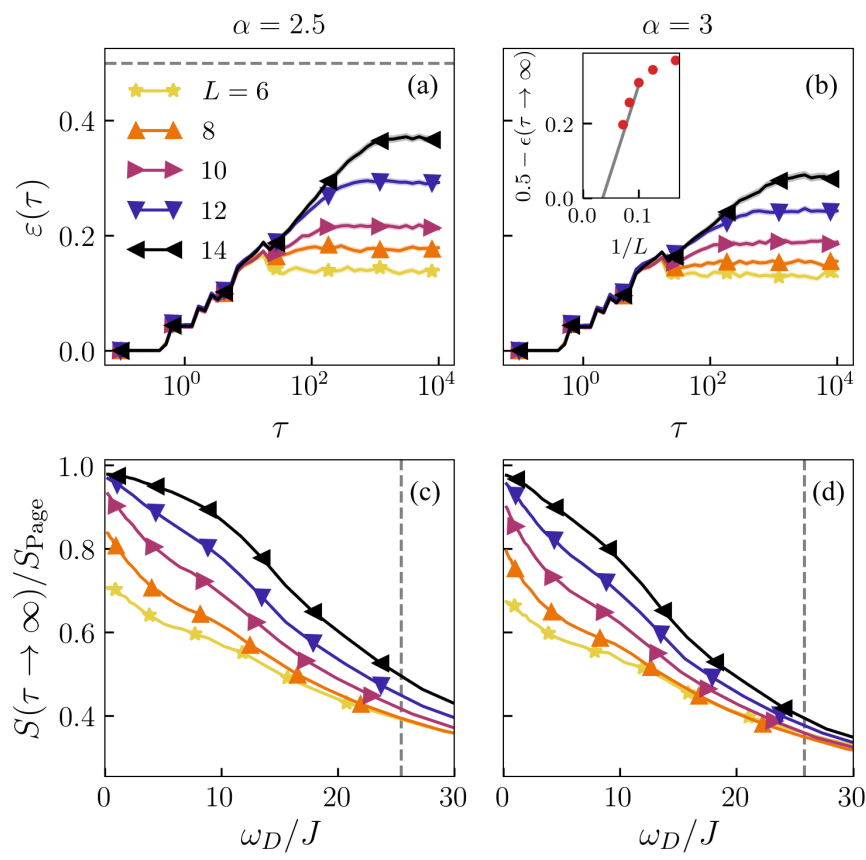

FIG. 8. In (a) and (b) are shown the energy absorption rate $\epsilon(\tau)$ in Eq. (4) starting from the ground state of the interacting model for two values of $\alpha$ for the interacting case. The inset in (b) shows the finite-size scaling for the long-time saturation value of $\epsilon(\tau)$. In (c) and (d) the long-time saturation point for the bipartite entanglement entropy with the initial state given by the Néel state $\left(\prod_{i}^{N} c_{2 i}^{\dagger}|0\rangle\right)$. The dashed vertical lines indicate the bandwidth $\Lambda(L)$.

Meanwhile at moderate frequencies $\delta S^{2}$ develops a growing peak with $L$, a feature which has been used as a fingerprint for the MBL transition [19,21]. However, as one can observe, the peak shifts inexorably with system size, getting closer to $\Lambda$.

Finally, we inspect some dynamical properties of the interacting driven system, i.e., energy and entanglement propagation.

As for the noninteracting case, we start with the propagation of energy. Figures 8(a) and 8(b) show the energy density $\epsilon(\tau)$ at moderate frequency $\omega_{D} \approx 4 J$. For both values, $\alpha=2.5,3$ and for our available system sizes, the infinitetemperature value $\left(\varepsilon=\frac{1}{2}\right)$ is not reached yet. However, in long-time limit, $\epsilon(\tau)$ tends to $\varepsilon \approx \frac{1}{2}$, which it also supported by the finite-size scaling (see inset in Fig. 8). The remaining two panels, Figs. 8(c) and 8(d), are dedicated to the asymptotic long-time saturation value of $S(\tau)$. As a function of the driving frequency, we compute $S(\tau \rightarrow \infty) / S_{\text {Page }}$, where $S(\tau \rightarrow \infty)$ is determined by averaging over a long-time window in which $S(\tau)$ reaches its saturation value. The numerical analysis of $S(\tau \rightarrow \infty) / S_{\text {Page }}$ in Figs. 8(c) and 8(d), in which is visible the tendency $S(\tau \rightarrow \infty) / S_{\text {Page }} \rightarrow 1$ as $L$ increases. Thus, in agreement with the results of the spectrum and eigenstates of $H_{\mathrm{F}}$, the finite-size analysis of $S(\tau \rightarrow \infty)$ further supports the conjecture that ergodicity is restored in the thermodynamic limit.

Unlike the case in which the LIOMs have exponentially decaying tails $(\alpha \rightarrow \infty)$, the algebraic MBL phase shows a clear tendency towards delocalization at any finite-driving frequencies. To better pin down this fundamental difference, 

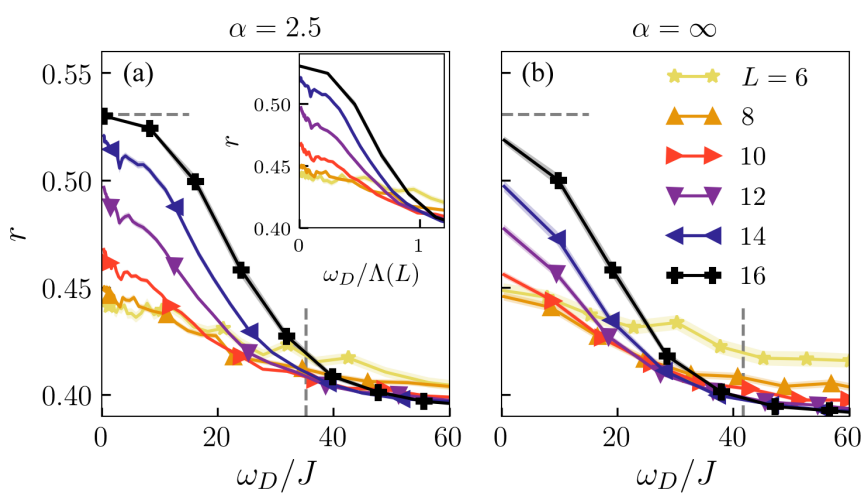

FIG. 9. $r$ statistics as a function of the driving frequency $\omega_{D}$, for the hopping decaying rate $\alpha=2.5$ in (a) and $\alpha \rightarrow \infty$ in (b). The dashed horizontal lines indicate the GOE $r$ value $\approx 0.531$ and the Poissonian one $\approx 0.386$, whereas the vertical lines indicate the spectral width $[\Lambda(L=16)]$. In the inset of (a) is shown the $r$ value as a function of $\omega_{D} / \Lambda(L)$ for $\alpha=2.5$. In both panels $W=6$ and $\delta=0.5$.

we study a Floquet minimal MBL model $[23,83,84]$, which can be considered as the strong disorder limit of $H$ in Eq. (1),

$$
U(T)=e^{-i H^{+} T / 2} e^{-i H^{-} T / 2},
$$

with

$$
H^{ \pm}=\sum_{l} \epsilon_{l}^{ \pm} I_{l}^{ \pm}+V \sum_{p, q} B_{p q}^{ \pm} I_{p}^{ \pm} I_{q}^{ \pm}
$$

where $\left\{\epsilon_{l}^{ \pm}\right\}$and $\left\{I_{l}^{ \pm}\right\}$are the single-particle energies and LIOMs of the noninteracting model $(V=0)$ in Eq. (1) with $\pm \delta$, respectively [23,83]. The couplings $B_{p q}=$ $\sum_{i}\left\{\left|\phi_{p}^{ \pm}(i)\right|^{2}\left|\phi_{q}^{ \pm}(i+1)\right|^{2}-\phi_{p}^{ \pm}(i) \phi_{p}^{ \pm}(i+1) \phi_{q}^{ \pm}(i) \phi_{q}^{ \pm}(i+1)\right\}$ are obtained using first-order perturbation theory in $H$ in the limit $V / W \ll 1[23,83]$. By construction, $H^{ \pm}$is localized meaning that it has Poissonian spectrum and integrals of motion are algebraically localized for $\alpha<\infty$ and exponential as $\alpha \rightarrow \infty$. As a further consequence, for $\alpha<\infty$ the couplings $B_{p q}$ decay algebraically with respect to the distance between the centers of localization of $\phi_{p}$ and $\phi_{q}$, while for $\alpha=\infty$ the decay is exponentially fast. The spatial structure of the decay of $B_{p q}$ determines the growth in time of the EE after a global quantum quench with $H^{ \pm}$. Thus, for $\alpha<\infty$ the EE has an unbounded algebraic growth, while for $\alpha=\infty$ the growth is logarithmically slow.

Returning back to the driven case, in Figs. 9(a) and 9(b) is shown the $r$ value as function of the driving frequency $\omega_{D}$ computed with the eigenvalues of the Floquet Hamiltonian of the minimal model in Eq. (15) for $\alpha=2.5$ (algebraically LIOMs) and $\alpha \rightarrow \infty$ (exponentially LIOMs). The crossing between curves for different system sizes, which may demarcate the existence of a putative MBL transition as function of $\omega_{D}$, is located in proximity of the many-body width cutoff for $\alpha=2.5$ and at smaller frequencies for $\alpha \rightarrow \infty$. Considering only values $\omega_{D} \leqslant \Lambda$ [see Fig. 9(a) and its inset], and the tendency towards delocalization for the algebraic case, we may conclude that the localization will be completely destroyed in the thermodynamic limit. Indeed, the crossing between curves for different $L$ happens for frequencies $\omega_{D} \geqslant \Lambda$. However, in the limiting case of exponential LIOMs $(\alpha \rightarrow \infty)$, we observe a much more robust and defined crossover between the two regimes which occurs before the natural bound $\omega_{D} \sim \Lambda(L)$, and therefore it may culminate to an MBL transition at a finite frequency strength $[27,28,34]$.

Now, we provide an analytical argument based on Ref. [34] to support the delocalization of an algebraic MBL phase under periodic drive. For the sake of simplicity, consider the case where the driving terms contain only of one harmonic and the time-dependent Hamiltonian can be separated in two parts $H(t)=H_{\ell \text {-bit }}(W)+V H_{1}(t)$. The Hamiltonian $H_{\ell \text {-bit }}(W)$ is algebraic MBL and $H_{1}(t) \sim \cos (2 \pi t / T)$ is the time-depended part which allows jumps between the localized eigenstates of $H_{\ell \text {-bit }}(W) . W$ and $V$ are the disorder and interaction strength, respectively. We assume that the disorder is strong enough to localize the instantaneous eigenstates. If $T$ is large enough, we can estimate the probability of mixing the instantaneous eigenstates $\left|E_{\alpha}(t)\right\rangle,\left|E_{\beta}(t)\right\rangle$ using the Landau-Zener formula [85-87]

$$
P_{\alpha \rightarrow \alpha}=e^{-C_{\alpha, \beta},} \quad C_{\alpha, \beta}=\pi \frac{\left|M_{\alpha, \beta}\right|^{2}}{v_{\alpha, \beta}},
$$

where $M_{\alpha, \beta}=\left\langle E_{\alpha}\left|H_{1}\left(t_{c}\right)\right| E_{\beta}\right\rangle$ is the matrix element coupling the eigenstates, the Landau-Zener velocity $v_{\alpha, \beta}=T \partial_{t}\left(E_{\alpha}-\right.$ $\left.E_{\beta}\right)\left.\right|_{t=t_{c}}$, and $t_{c}$ the time at which the crossing takes place. In the adiabatic $\left(C_{\alpha, \beta} \approx 0\right)$ and diabatic $\left(C_{\alpha, \beta} \gg 1\right)$ limits the system remains localized after the crossing. In the intermediate situation eigenstates are characterized by different values of LIOMs mixing together and starting to thermalize. As argued in Ref. [34], there exist two length scales $x_{1}$ and $x_{2}$, which determine the behavior of $P_{\alpha \rightarrow \alpha}$. Consider two eigenstates $\left|E_{\alpha}(t)\right\rangle,\left|E_{\beta}(t)\right\rangle$ which differ from the values of LIOMs only in a region of size $x$. Thus, these eigenstates are the same outside a region of size $x$. There are $\sim 2^{x}$ of these eigenstates which typically belong to an energy shell with bandwidth $\sim W \sqrt{x}$ and the mean level spacing

$$
\Delta(x)=W \sqrt{x} / 2^{x} .
$$

On the other hand, the typical gap at the crossing is controlled by $V H_{1}\left(t_{c}\right)$ :

$$
\delta E_{\alpha, \beta}(x) \sim V\left(\left\langle E_{\alpha}\left|H_{1}\right| E_{\alpha}\right\rangle-\left\langle E_{\beta}\left|H_{1}\right| E_{\beta}\right\rangle\right) \sim V \sqrt{x} .
$$

In the limit $\delta E_{\alpha, \beta} \gg \Delta$ multiple crossing between eigenstates which differ only in the region of size $x$ happen. This sets the length scale $x_{1}$ by comparing $\delta E_{\alpha, \beta}\left(x_{1}\right) \sim \Delta\left(x_{1}\right)$ :

$$
x_{1}=\log _{2} \frac{W}{V} .
$$

The length $x_{2}$ determinants the character of the transition, which is directly connected to $C_{\alpha, \beta} \propto\left|M_{\alpha, \beta}\right|^{2} / v_{\alpha, \beta}$. The matrix element between two algebraically localized states can be estimated by

$$
M_{\alpha, \beta} \sim V / x^{\gamma},
$$

which decays as a power law in distance $x$ since the LIOMs are algebraically localized. Instead, the Landau-Zener velocity is

$$
v_{\alpha, \beta} \sim \Delta E_{\alpha, \beta} / T \sim V \sqrt{x} / T .
$$


Thus, the length $x_{2}$ is defined by $C_{\alpha, \beta}\left(x_{2}\right) \sim 1$ which separate the adiabatic limit from the diabatic one,

$$
x_{2}=(V T)^{\frac{1}{2 \gamma+1 / 2}} .
$$

In the regime $x_{1} / x_{2} \ll 1$, the system undergoes multiples crossing in an intermediate regime $C_{\alpha, \beta} \sim 1$ which implies the thermalization of the region of size $x \ll x_{1} \ll x_{2}$. The relation $x_{1} / x_{2} \ll 1$ implies that

$$
\frac{W}{V} \ll 2^{(V T)^{\frac{1}{2 y+1 / 2}}} .
$$

Although this simple argument [Eq. (23)] does not strictly prove the delocalization of the system at any frequency $\omega_{D}=$ $\frac{2 \pi}{T}$, it shows that the delocalization is parametrically favorable, meaning that it is exponentially sensitive to $\omega_{D} \sim 1 / T$.

\section{CONCLUSION}

In this work we have investigated the stability of an algebraically localized phase subject to periodically global driving for both the noninteracting and the interacting cases. For sufficiently strong disorder, the noninteracting algebraically localized phase is robust once short-range interactions are turned on, generalizing the paradigm of algebraic localization to the interacting case (algebraic MBL). As a consequence, in an algebraic MBL phase, ergodicity is broken and the phase is fully characterized by an extensive number of integrals of motion with algebraic decaying tails, which are believed to be adiabatically connected to the algebraic decaying integrals of motion of the noninteracting case.

In particular, we studied a periodically driven (Floquet) spinless fermionic chain with long-range random hopping and density-density short-range interactions. The nondriven noninteracting limit is known as the power-law random banded matrix model and hosts an algebraically localized phase, meaning that its single-particle eigenstates are localized with power-law tails.

First, we considered the noninteracting case. With a combination of analytical arguments and exact numerical simulations, we provided evidence that the algebraically localized phase is stable under driving. The spectrum of the singleparticle Floquet Hamiltonian shows Poissonian statistics and its eigenstates are localized. We supported this claim by using analytical arguments based on strong disorder renormalization group techniques.

Second, we have tackled the interacting case by turning on short-range density-density type interactions. At strong disorder and for sufficiently large decay rate of the hopping the nondriven case is believed to be in an algebraic MBL phase. Several MBL markers have been used to numerically inspect the stability of the algebraic MBL phase under driving. After a careful analysis of the finite-size effects, we argue that ergodicity might be restored in the thermodynamic limit for any driving frequencies. For finite-size systems we showed that localization sets in for driving frequency that are comparable with the many-body bandwidth. This indicates that the finite-size localized phase might disperse in the thermodynamic limit. Analytical arguments were made to support our numerical results by showing that delocalization appears parametrically favorable.

Thus, unlike the noninteracting case, we showed that the algebraic MBL phase is unstable once the system is periodically driven. This result should be compared to the case in which the LIOMs are exponentially localized. For conventional MBL phases, it is believed that a finite, critical frequency exists, which separates an ergodic phase from an MBL one. To further point out this difference, in the final part of the work we inspected a toy Floquet model, in which we can tune the type of localization, from algebraic to exponential. We confirmed the existence of a critical driving frequency in a conventional MBL phase and showed the breakdown of an algebraic MBL phase under driving.

Our work imposes strong constraints on the possible existence of a genuine MBL phase for long-range models under driving. This result could be relevant for trapped-ion experiments, where long-range interactions are present. In particular, our work implies the absence of an MBL discrete time crystal phase for long-range models studied experimentally in Refs. [32,44] (see Refs. [42,43] for a recent discussions). It is important to point out that even though we do not expect the existence of an MBL phase, interesting nonergodic behavior could persist for fairly long timescales and large systems. Thus, understanding the timescales for thermalization is an important question which is left for future research.

\section{ACKNOWLEDGMENTS}

We would like to thank S. Bera and I. Khaymovich for helpful discussions. We also express our gratitude to $\mathrm{S}$. Woo Kim for a critical reading of the manuscript. This project has received funding from the European Research Council (ERC) under the European Union's Horizon 2020 research and innovation program (Grant Agreement No. No. 853443), and M.H. further acknowledges support by the Deutsche Forschungsgemeinschaft (DFG) via the Gottfried Wilhelm Leibniz Prize program. G.D.T. acknowledges the support from the EPiQS Program of the Gordon and Betty Moore Foundation.
[1] I. Bloch, J. Dalibard, and W. Zwerger, Many-body physics with ultracold gases, Rev. Mod. Phys. 80, 885 (2008).

[2] C. Gross and I. Bloch, Quantum simulations with ultracold atoms in optical lattices, Science 357, 995 (2017).

[3] D. A. Abanin, E. Altman, I. Bloch, and M. Serbyn, Colloquium: Many-body localization, thermalization, and entanglement, Rev. Mod. Phys. 91, 021001 (2019).
[4] R. Nandkishore and D. A. Huse, Many-body localization and thermalization in quantum statistical mechanics, Annu. Rev. Condens. Matter Phys. 6, 15 (2015).

[5] F. Alet and N. Laflorencie, Many-body localization: An introduction and selected topics, C. R. Phys. 19, 498 (2018).

[6] D. Basko, I. Aleiner, and B. Altshuler, Metal-insulator transition in a weakly interacting many-electron system with localized single-particle states, Ann. Phys. 321, 1126 (2006). 
[7] M. Serbyn, Z. Papić, and D. A. Abanin, Local Conservation Laws and the Structure of the Many-Body Localized States, Phys. Rev. Lett. 111, 127201 (2013).

[8] D. A. Huse, R. Nandkishore, and V. Oganesyan, Phenomenology of fully many-body-localized systems, Phys. Rev. B 90, 174202 (2014).

[9] J. Z. Imbrie, On many-body localization for quantum spin chains, J. Stat. Phys. 163, 998 (2016).

[10] V. Ros, M. Müller, and A. Scardicchio, Integrals of motion in the many-body localized phase, Nucl. Phys. B 891, 420 (2015).

[11] J. H. Bardarson, F. Pollmann, and J. E. Moore, Unbounded Growth of Entanglement in Models of Many-Body Localization, Phys. Rev. Lett. 109, 017202 (2012).

[12] M. Žnidarič, T. Prosen, and P. Prelovšek, Many-body localization in the heisenberg $x x z$ magnet in a random field, Phys. Rev. B 77, 064426 (2008).

[13] M. Serbyn, Z. Papić, and D. A. Abanin, Universal Slow Growth of Entanglement in Interacting Strongly Disordered Systems, Phys. Rev. Lett. 110, 260601 (2013).

[14] J. M. Deutsch, Quantum statistical mechanics in a closed system, Phys. Rev. A 43, 2046 (1991).

[15] M. Srednicki, Chaos and quantum thermalization, Phys. Rev. E 50, 888 (1994).

[16] M. Rigol, V. Dunjko, and M. Olshanii, Thermalization and its mechanism for generic isolated quantum systems, Nature (London) 452, 854 (2008).

[17] L. D'Alessio, Y. Kafri, A. Polkovnikov, and M. Rigol, From quantum chaos and eigenstate thermalization to statistical mechanics and thermodynamics, Adv. Phys. 65, 239 (2016).

[18] A. Pal and D. A. Huse, Many-body localization phase transition, Phys. Rev. B 82, 174411 (2010).

[19] J. A. Kjäll, J. H. Bardarson, and F. Pollmann, Many-Body Localization in a Disordered Quantum Ising Chain, Phys. Rev. Lett. 113, 107204 (2014).

[20] S. Bera, H. Schomerus, F. Heidrich-Meisner, and j. H. Bardarson, Many-Body Localization Characterized from a OneParticle Perspective, Phys. Rev. Lett. 115, 046603 (2015).

[21] D. J. Luitz, N. Laflorencie, and F. Alet, Many-body localization edge in the random-field heisenberg chain, Phys. Rev. B 91, 081103(R) (2015).

[22] G. De Tomasi, S. Bera, J. H. Bardarson, and F. Pollmann, Quantum Mutual Information as a Probe for Many-Body Localization, Phys. Rev. Lett. 118, 016804 (2017).

[23] G. De Tomasi, Algebraic many-body localization and its implications on information propagation, Phys. Rev. B 99, 054204 (2019).

[24] T. Botzung, D. Vodola, P. Naldesi, M. Müller, E. Ercolessi, and G. Pupillo, Algebraic localization from power-law couplings in disordered quantum wires, Phys. Rev. B 100, 155136 (2019).

[25] X. Deng, G. Masella, G. Pupillo, and L. Santos, Universal Algebraic Growth of Entanglement Entropy in Many-Body Localized Systems with Power-Law Interactions, Phys. Rev. Lett. 125, 010401 (2020).

[26] A. Safavi-Naini, M. L. Wall, O. L. Acevedo, A. M. Rey, and R. M. Nandkishore, Quantum dynamics of disordered spin chains with power-law interactions, Phys. Rev. A 99, 033610 (2019).
[27] A. Lazarides, A. Das, and R. Moessner, Fate of Many-Body Localization under Periodic Driving, Phys. Rev. Lett. 115, 030402 (2015).

[28] P. Ponte, Z. Papić, F. Huveneers, and D. A. Abanin, Many-Body Localization in Periodically Driven Systems, Phys. Rev. Lett. 114, 140401 (2015).

[29] F. Wilczek, Quantum Time Crystals, Phys. Rev. Lett. 109, 160401 (2012).

[30] K. Sacha, Modeling spontaneous breaking of time-translation symmetry, Phys. Rev. A 91, 033617 (2015).

[31] D. V. Else, B. Bauer, and C. Nayak, Floquet Time Crystals, Phys. Rev. Lett. 117, 090402 (2016).

[32] N. Y. Yao, A. C. Potter, I.-D. Potirniche, and A. Vishwanath, Discrete Time Crystals: Rigidity, Criticality, and Realizations, Phys. Rev. Lett. 118, 030401 (2017).

[33] V. Khemani, A. Lazarides, R. Moessner, and S. L. Sondhi, Phase Structure of Driven Quantum Systems, Phys. Rev. Lett. 116, 250401 (2016).

[34] D. A. Abanin, W. DeRoeck, and F. Huveneers, Theory of manybody localization in periodically driven systems, Ann. Phys. 372, 1 (2016).

[35] P. Bordia, H. Lüschen, U. Schneider, M. Knap, and I. Bloch, Periodically driving a many-body localized quantum system, Nat. Phys. 13, 460 (2017).

[36] X. Deng, V. E. Kravtsov, G. V. Shlyapnikov, and L. Santos, Duality in Power-Law Localization in Disordered One-Dimensional Systems, Phys. Rev. Lett. 120, 110602 (2018).

[37] P. A. Nosov, I. M. Khaymovich, and V. E. Kravtsov, Correlation-induced localization, Phys. Rev. B 99, 104203 (2019).

[38] L. S. Levitov, Absence of localization of vibrational modes due to dipole-dipole interaction, Europhys. Lett. 9, 83 (1989).

[39] A. D. Mirlin and Y. V. Fyodorov, The statistics of eigenvector components of random band matrices: analytical results, J. Phys. A: Math. Gen. 26, L551 (1993).

[40] L. S. Levitov, Delocalization of Vibrational Modes Caused by Electric Dipole Interaction, Phys. Rev. Lett. 64, 547 (1990).

[41] A. D. Mirlin, Y. V. Fyodorov, F.-M. Dittes, J. Quezada, and T. H. Seligman, Transition from localized to extended eigenstates in the ensemble of power-law random banded matrices, Phys. Rev. E 54, 3221 (1996).

[42] V. Khemani, R. Moessner, and S. L. Sondhi, A comment on "Discrete time crystals: Rigidity, criticality, and realizations", arXiv:2109.00551.

[43] N. Y. Yao, A. C. Potter, I.-D. Potirniche, and A. Vishwanath, A comment on "Discrete time crystals: Rigidity, criticality, and realizations", arXiv:2109.07485.

[44] J. Zhang, P. W. Hess, A. Kyprianidis, P. Becker, A. Lee, J. Smith, G. Pagano, I.-D. Potirniche, A. C. Potter, A. Vishwanath, N. Y. Yao, and C. Monroe, Observation of a discrete time crystal, Nature 543, 217 (2017).

[45] M. C. Tran, A. Ehrenberg, A. Y. Guo, P. Titum, D. A. Abanin, and A. V. Gorshkov, Locality and heating in periodically driven, power-law-interacting systems, Phys. Rev. A 100, 052103 (2019).

[46] V. Kravtsov, I. Khaymovich, E. Cuevas, and M. Amini, A random matrix model with localization and ergodic transitions, New J. Phys. 17, 122002 (2015). 
[47] V. L. Quito, P. Titum, D. Pekker, and G. Refael, Localization transition in one dimension using wegner flow equations, Phys. Rev. B 94, 104202 (2016).

[48] E. Khatami, M. Rigol, A. Relano, and A. M. García-García, Quantum quenches in disordered systems: Approach to thermal equilibrium without a typical relaxation time, Phys. Rev. E 85, 050102(R) (2012).

[49] T. Thiery, F. M. c. Huveneers, M. Muller, and W. DeRoeck, Many-Body Delocalization as a Quantum Avalanche, Phys. Rev. Lett. 121, 140601 (2018).

[50] S. Gopalakrishnan and D. A. Huse, Instability of many-body localized systems as a phase transition in a nonstandard thermodynamic limit, Phys. Rev. B 99, 134305 (2019).

[51] W. DeRoeck and F. M. c. Huveneers, Stability and instability towards delocalization in many-body localization systems, Phys. Rev. B 95, 155129 (2017).

[52] T. B. Wahl, A. Pal, and S. H. Simon, Signatures of the manybody localized regime in two dimensions, Nat. Phys. 15, 164 (2019).

[53] W. DeRoeck and J. Z. Imbrie, Many-body localization: stability and instability, Philos. Trans. R. Soc. A 375, 20160422 (2017).

[54] D. M. Kennes, Many-body localization in two dimensions from projected entangled-pair states, arXiv:1811.04126.

[55] H. Theveniaut, Z. Lan, G. Meyer, and F. Alet, Transition to a many-body localized regime in a two-dimensional disordered quantum dimer model, Phys. Rev. Research 2, 033154 (2020).

[56] I.-D. Potirniche, S. Banerjee, and E. Altman, Exploration of the stability of many-body localization in $d>1$, Phys. Rev. B 99, 205149 (2019).

[57] E. Chertkov, B. Villalonga, and B. K. Clark, Numerical Evidence for Many-Body Localization in Two and Three Dimensions, Phys. Rev. Lett. 126, 180602 (2021).

[58] F. Pietracaprina and F. Alet, Probing many-body localization in a disordered quantum dimer model on the honeycomb lattice, SciPost Phys. 10, 44 (2021).

[59] S. J. Thomson and M. Schiró, Time evolution of many-body localized systems with the flow equation approach, Phys. Rev. B 97, 060201(R) (2018).

[60] H.-K. Tang, N. Swain, D. C. W. Foo, B. J. J. Khor, F. F. Assaad, S. Adam, and P. Sengupta, Evidence of many-body localization in 2D from quantum Monte Carlo simulation, arXiv:2106.08587.

[61] K. S. C. Decker, D. M. Kennes, and C. Karrasch, Manybody localization and the area law in two dimensions, arXiv:2106.12861.

[62] N. Y. Yao, C. R. Laumann, S. Gopalakrishnan, M. Knap, M. Müller, E. A. Demler, and M. D. Lukin, Many-Body Localization in Dipolar Systems, Phys. Rev. Lett. 113, 243002 (2014).

[63] A. L. Burin, Localization in a random xy model with longrange interactions: Intermediate case between single-particle and many-body problems, Phys. Rev. B 92, 104428 (2015).

[64] S. Gopalakrishnan and S. Parameswaran, Dynamics and transport at the threshold of many-body localization, Phys. Rep. 862, 1 (2020), dynamics and transport at the threshold of many-body localization.

[65] K. S. Tikhonov and A. D. Mirlin, Many-body localization transition with power-law interactions: Statistics of eigenstates, Phys. Rev. B 97, 214205 (2018).

[66] A. O. Maksymov and A. L. Burin, Many-body localization in spin chains with long-range transverse interactions: Scaling of critical disorder with system size, Phys. Rev. B 101, 024201 (2020).

[67] J.-y. Choi, S. Hild, J. Zeiher, P. Schauß, A. Rubio-Abadal, T. Yefsah, V. Khemani, D. A. Huse, I. Bloch, and C. Gross, Exploring the many-body localization transition in two dimensions, Science 352, 1547 (2016).

[68] J. Smith, A. Lee, P. Richerme, B. Neyenhuis, P. W. Hess, P. Hauke, M. Heyl, D. A. Huse, and C. Monroe, Many-body localization in a quantum simulator with programmable random disorder, Nat. Phys. 12, 907 (2016).

[69] K. Xu, J.-J. Chen, Y. Zeng, Y.-R. Zhang, C. Song, W. Liu, Q. Guo, P. Zhang, D. Xu, H. Deng, K. Huang, H. Wang, X. Zhu, D. Zheng, and H. Fan, Emulating Many-Body Localization with a Superconducting Quantum Processor, Phys. Rev. Lett. 120, 050507 (2018).

[70] Q. Guo, C. Cheng, H. Li, S. Xu, P. Zhang, Z. Wang, C. Song, W. Liu, W. Ren, H. Dong, R. Mondaini, and H. Wang, Stark many-body localization on a superconducting quantum processor, arXiv:2011.13895.

[71] G. De Tomasi, I. M. Khaymovich, F. Pollmann, and S. Warzel, Rare thermal bubbles at the many-body localization transition from the Fock space point of view, Phys. Rev. B 104, 024202 (2021).

[72] P. T. Dumitrescu, A. Goremykina, S. A. Parameswaran, M. Serbyn, and R. Vasseur, Kosterlitz-thouless scaling at manybody localization phase transitions, Phys. Rev. B 99, 094205 (2019).

[73] V. Oganesyan and D. A. Huse, Localization of interacting fermions at high temperature, Phys. Rev. B 75, 155111 (2007).

[74] Y. Y. Atas, E. Bogomolny, O. Giraud, and G. Roux, Distribution of the Ratio of Consecutive Level Spacings in Random Matrix Ensembles, Phys. Rev. Lett. 110, 084101 (2013).

[75] D. N. Page, Average Entropy of a Subsystem, Phys. Rev. Lett. 71, 1291 (1993).

[76] R. Singh, R. Moessner, and D. Roy, Effect of longrange hopping and interactions on entanglement dynamics and many-body localization, Phys. Rev. B 95, 094205 (2017).

[77] F. Evers and A. D. Mirlin, Anderson transitions, Rev. Mod. Phys. 80, 1355 (2008).

[78] S. Kehrein, The Flow Equation Approach to Many-particle Systems, Vol. 217 (Springer, Berlin, 2007).

[79] F. Wegner, Flow-equations for Hamiltonians, Ann. Phys. 506, 77 (1994).

[80] S. D. Głazek and K. G. Wilson, Renormalization of Hamiltonians, Phys. Rev. D 48, 5863 (1993).

[81] S. D. Glazek and K. G. Wilson, Perturbative renormalization group for Hamiltonians, Phys. Rev. D 49, 4214 (1994).

[82] D. Pekker, B. K. Clark, V. Oganesyan, and G. Refael, Fixed Points of Wegner-Wilson Flows and Many-Body Localization, Phys. Rev. Lett. 119, 075701 (2017).

[83] G. De Tomasi, F. Pollmann, and M. Heyl, Efficiently solving the dynamics of many-body localized systems at strong disorder, Phys. Rev. B 99, 241114(R) (2019).

[84] L.-N. Wu, A. Schnell, G. D. Tomasi, M. Heyl, and A. Eckardt, Describing many-body localized systems in thermal environments, New J. Phys. 21, 063026 (2019).

[85] C. Zener, Nonadiabatic crossing of energy levels, Proc. R. Soc. London A 137, 696 (1932). 
[86] Theorie der unelastischen stösse zwischen atomen, in E.C.G. Stueckelberg, An Unconventional Figure of Twentieth Century Physics: Selected Scientific Papers with Commentaries, edited by J. Lacki, H. Ruegg, and G. Wanders, (Birkhäuser, Basel, 2009), pp. 117-171.
[87] E. Majorana, Atomi orientati in campo magnetico variabile, Il Nuovo Cimento (1924-1942) 9, 43 (1932).

[88] G. De Tomasi and I. M. Khaymovich, Multifractality Meets Entanglement: Relation for Nonergodic Extended States, Phys. Rev. Lett. 124, 200602 (2020). 